\title{
CORRECCIONES DIFERENCIALES VÍA NTRIP PARA DISPOSITIVO MÓVIL CON APLICACIÓN ANDROID
}

\section{Differential corrections by NTRIP to mobile devices with android application}

\author{
ALFONSO TIERRA \\ OSCAR SILVA \\ CÉSAR LEIVA
}

Grupo de Investigación Geoespacial. Universidad de las Fuerzas Armadas-ESPE Av. Gral Rumiñahuis/n - Sangolquí, Ecuador

P.O.Box 171-5-31B

e-mail: oscarfsv@gmail.com; artierra@espe.edu.ec; leicesar@hotmail.com

\section{RESUMEN}

El posicionamiento en tiempo real usando GPS se hace mediante correcciones diferenciales enviadas desde una estación base hacia un receptor móvil. Generalmente, la corrección es enviada vía radio o por medio de satélites de comunicación. Con el desarrollo del internet inalámbrica y con el aumento de la cobertura de la señal mediante un Protocolo, conocido como IP, han hecho que se integren estas tecnologías para realizar mediciones para trabajos y estudios en tiempo real usando la tecnología GPS, y mediante el protocolo NTRIP. Esta técnica se ha estado desarrollando e implementando de forma constante en la región, siendo el presente y futuro de las mediciones para trabajos de precisión y en tiempo real. En este trabajo, se muestra la implementación de la técnica NTRIP para posicionamiento en tiempo real. El acceso a datos brutos del GPS en un móvil ANDROID y un navegador Mobile Mapper 10, la utilización de NTRIP para el envió de correcciones diferenciales por medio del protocolo IP, y el internet como red de información inmediata, fueron la base general para crear una aplicación que utiliza una corrección diferencial por posición. Los primeros resultados muestran una mejoría en la posición, pudiendo llegar a obtener un error medio de $2.3 \mathrm{~m}$ en una Tablet con Android, y $1.6 \mathrm{~m}$ con el Mobile Mapper 10; en un tiempo de 5 minutos. Palabras clave: GNSS; NTRIP; Android; Código C/A; Corrección Diferencial. 


\section{RESUMO}

O posicionamento em tempo real usando GPS é feito por meio de correções diferenciais desde uma base até um receptor móvel. Geralmente, a correção é feita via rádio ou por meio de satélites de comunicação. Com o desenvolvimento da internet sem fio e o aumento da cobertura do sinal através de um protocolo conhecido como IP tem-se intensificado os trabalhos em tempo real integrando o GPS e o NTRIP. Esta técnica esta sendo desenvolvida em algumas regiões chegando ser o presente e o futuro dos trabalhos de precisão em tempo real. Neste trabalho é mostrado a implementação da técnica NTRIP para o posicionamento em tempo real. $\mathrm{O}$ acesso aos dados do GPS num navegador móvel com Android e no Mobile Mapper 10, o uso de NTRIP para fazer as correcões diferenciais por meio do IP, e a internet como meio de comunicação, foram as bases para fazer a aplicação das correções na posição e melhorar o posicionamento do móvel. Os primeiros resultados mostram que o erro médio posicionamento do móvel depois de fazer as correções pode chegar até $2.3 \mathrm{~m}$ em uma Tablet com Android, and $1.6 \mathrm{~m}$ com o Mobile Mapper 10; em de 5 minutos de rastreio.

Palavras chave: GPS; NTRIP; Android; Correção Diferencial.

\section{ABSTRACT}

Real time GPS positioning is obtained by means of differential corrections sent from a base station to a mobile receiver. Usually, the correction is sent via radio or also by means of communication satellites. With the internet development and with the increase in the signal coverage thanks to a protocol, the GPS technology with NTRIP protocol are now used for studies in real-time. This technique is being developed and implemented in many regions, and it actually is the present and future of precision measurement work in real time. In this study, the implementation of the NTRIP technique for positioning in real time is described. The access to raw data from the GPS on a mobile Android and a Mobile Mapper 10, the use of NTRIP for sending differential corrections using the IP protocol, and the internet as an information network, were the mechanisms and tools used for creating a differential correction by position application. The first results show an improvement in the position determination which may achieve mean error of $2.3 \mathrm{~m}$ in the Tablet with Android, and 1.6m with the Mobile Mapper 10, in a time lapse of 5 minutes.

Keywords: GPS; NTRIP; Android; Differential Correction.

\section{INTRODUCCIÓN}

La disponibilidad de teléfonos inteligentes con la capacidad de recibir señales GPS (Global Positioning Systems) y poder navegar en cualquier parte del mundo es inmensa, trabaja con código C/A que es de libre acceso y tiene errores en metros. Por otro lado, la disponibilidad de estaciones GNSS (Global Navigation Satellite System) o Estaciones de Monitoreo Continuo-EMC, se ha convertido en la 
herramienta indispensable para trabajos con precisión, además de tener la capacidad de generar correcciones diferenciales de código y fase.

Los métodos y procedimientos para el cálculo de posiciones en todo el planeta, a lo largo de la historia, han ido evolucionando con la finalidad de encontrar mejores precisiones, disminuir los errores y mejorar la confiabilidad de los resultados obtenidos en un procesamiento de datos GNSS.

Actualmente, existen muchos equipos que pueden utilizarse en cualquier tipo de ambiente y condiciones, hardware y software que trabajan en forma simultánea, arrojando datos de alta precisión, en la mayoría de los casos son trabajados en postproceso para obtener un mejor resultado.

La necesidad de tener equipos de posicionamiento que respondan con datos en tiempo real, han llevado a los investigadores a desarrollar mecanismos y técnicas. Éstos, unidos con la tecnología actual de redes de información inmediata, han podido formar un conjunto de métodos, que se han ido desarrollando progresivamente, para tener información en tiempo real y con una alta confiabilidad en el posicionamiento. Los equipos GNSS o estaciones de monitoreo continuo, se han convertido en la herramienta ideal para realizar trabajos con precisión y enviar información en tiempo real, como correcciones diferenciales de código y fase.

La disponibilidad de dispositivos móviles con capacidad de recibir señales GPS y poder navegar en cualquier parte del mundo es inmensa y cada vez a menor costo. La limitante de estos dispositivos es su precisión que puede alcanzar, puesto que solo recibe la señal de código C/A que es de libre acceso y tiene errores en metros.

En el año 2002 en Alemania, el NTRIP (Network Transportation of RTCM via Internet Protocol) fue desarrollado por la BKG (Bundesamt für Kartographie und Geodäsie) y consistió en transmitir correcciones diferenciales GNSS a través del protocolo de internet (TCP/ IP) originalmente en formato estándar RTCM (The Radio Technical Commission for Maritime Services), por medio del protocolo de transferencia de hipertexto (HTTP), dichas correcciones son calculadas desde la estación de referencia y ofrecidas a los usuarios vía internet. (HOYER et.al. 2010). La ventaja del uso del NTRIP consistió en la estandarización de los foramatos de los datos. Antes de esto, cada fabricante de un equipo tenía su propio estándar de formato para aplicaciones en tiempo real.

Posteriormente, en varios países se han realizado estudios relacionados con la tecnología NTRIP, como es el caso de los países que integran SIRGAS, como por ejemplo en Venezuela (MÁRQUEZ, 2007), Brasil ( COSTA, et.al., 2009), Argentina (PIÑON\&CIMBARO, 2010), Uruguay (YELICICH, et.al., 2013), Ecuador (SILVA, et.al, 2013), entre otros.

El objetivo de este trabajo es realizar las correcciones diferenciales desde una EMC base, hacia un dispositivo móvil mediante la técnica NTRIP, para lo cual se utilizó una Tablet con sistema Android, capacidad de recibir el código C/A, y la programación de un interface de aplicación. También, se utilizó el receptor Mobile 
Maper 10 como navegador, para recibir las correcciones diferenciales con la finalidad de comparar los resultados.

\section{POSICIONAMIENTO CINEMÁTICO CONTINUO}

De los diferentes métodos de posicionamiento que se pueden ejecutar con GPS, se encuentran los métodos rápidos conocidos como cinemáticos, pudiendo sus coordenadas ser determinadas ya sea en post-proceso o en tiempo real (SEEBER, 1993), (TORGE \& MULLER, 2012). Es un método diferencial entre dos receptores, uno actuando como base y el otro como móvil. El móvil para mejorar su posicionamiento recibe correcciones en tiempo real desde la base. Estas correcciones pueden ser realizadas a las coordenadas o a las falsas distancias, y recibidas vía radio, satélites de comunicaciones, u otros medios o plataformas de telecomunicaciones. Actualmente, con el mejoramiento de la cobertura del Internet inalámbrica, ha hecho que se use como medio de transmisión de las correcciones vía IP (Internet Protocol) desde la base al móvil.

\subsection{Networked Transport of RTCM Via Internet Protocol - NTRIP}

Consiste en la transmisión de correcciones diferenciales GNSS (Global Navigation Satellite System, originalmente en formato estándar RTCM(Radio Technical Comission for Marine Sevices) (RTCM, 2001), (FERNÁNDEZ, 2008), a través del protocolo de transferencia de hipertexto (HTTP - Hipertext Transfer Protocol), (NILSSON,2007), las cuales son calculadas desde una estación de referencia o base, y ofrecidas a los usuarios vía Internet.

Como se puede observar en la Figura 1. Este sistema NTRIP esa constituido fundamentalmente por los siguientes componentes: NTRIPSource, NRIPServer, NTRIPCaster y NTRIPClient. (BKG, 2005).

Figura 1 - Arquitectura NTRIP. Fuente: (MÁRQUEZ, 2007).

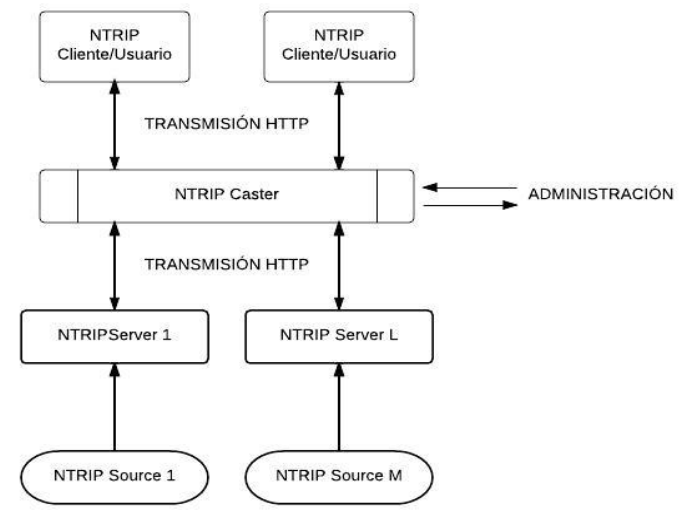

Bol. Ciênc. Geod., sec. Artigos, Curitiba, v. 21, nº 1, p.43-55, jan-mar, 2015. 
Basado en una transmisión estándar HTTP relativamente fácil de implementar cuando se tiene cliente y los recursos limitados de servidores de plataforma disponibles. La aplicación no se limita a un contenido particular claro o codificado, tiene la capacidad para distribuir cualquier tipo de dato GNSS. Además, tiene un potencial para apoyar el uso masivo; con difusión de cientos de señales simultáneas, de miles de usuarios posibles en la aplicación de Radio modificado, la radiodifusión en Internet del software.

El protocolo fue desarrollado para que pueda ser usado en una difusión de cientos de streams, conexiones masivas y simultaneas de usuarios, ya que el CASTERNTRIP es un equipo (computadora) de distribución de señales para difusión en internet.

\subsection{Android y GPS}

El uso de esta técnica, permite al usuario tener coordenadas GPS en tiempo real, sin necesidad de utilizar el post- proceso para mejorar su posición. Así mismo, la tecnología inalámbrica se ha ido implementando poco a poco en diferentes usuarios. A pesar que, el internet es público en muchos lugares por medio de la red Wi-Fi, no llega a abarcar un área extensa, llevando a desarrollar redes de comunicación para el internet de $2 \mathrm{G}, 3 \mathrm{G}$ y $4 \mathrm{G}$. Este último con una gran velocidad de navegación tanto de descarga como de subida de información, sin embargo este servicio, para la utilización de NTRIP, tiene un costo adicional.

Los dispositivos móviles, como teléfonos celulares, tablet, smartphones han tenido un gran desarrollo tecnológico, haciéndose cada vez más accesible y de fácil uso, permitiendo el aumento de diferentes aplicaciones. Dependiendo del fabricante los dispositivos cuentan con su sistema operativo propio como por ejemplo el Windows Mobile, IOS, Android.

Android es una plataforma móvil, que incluye un sistema operativo enfocado para ser utilizado en varios dispositivos móviles como el caso de la Tablet. Este sistema operativo proporciona todas las interfaces necesarias para desarrollar nuevas aplicaciones en una forma sencilla y en un lenguaje basado en Java (Báez, et.al., 2010). La característica más importante es que se trata de una plataforma totalmente abierta, tanto para fabricantes de dispositivos móviles como para desarrolladores de aplicaciones

Generalmente, en una Tablet se dispone de un GPS integrado y con sistema Android, pudiendo acceder al servicio de navegación por satélites, el cual permite hacer geolocalización en tiempo real. En la Figura 2, se muestra su estructura interna y sus componentes. satélites.

GPS Chip. Es un receptor de radio frecuencia que se comunica con los

GPS Driver. Usa API (Application Programming Interface) de bajo nivel para que haya una comunicación con el chip GPS.

GL Engine. Recoge la información de localización de las torres móviles y lee el NVRAM (Chip que guarda la información o contenido cuando la batería esta 
baja, compuesto de una combinación de chips SRAM y EEPROM.). Capta múltiples satélites visibles, asiste al GPS Driver, fija la posición con información adicional transmitida desde satélites o accede vía internet a los servidores de red SUPL/NTP y de ser necesario, guarda la información en NVRAM para un uso posterior, inicializando el GPS con datos ya registrados.

Figura 2 - Estructura del GPS en Android.

Fuente: (LATORRE, 2012).

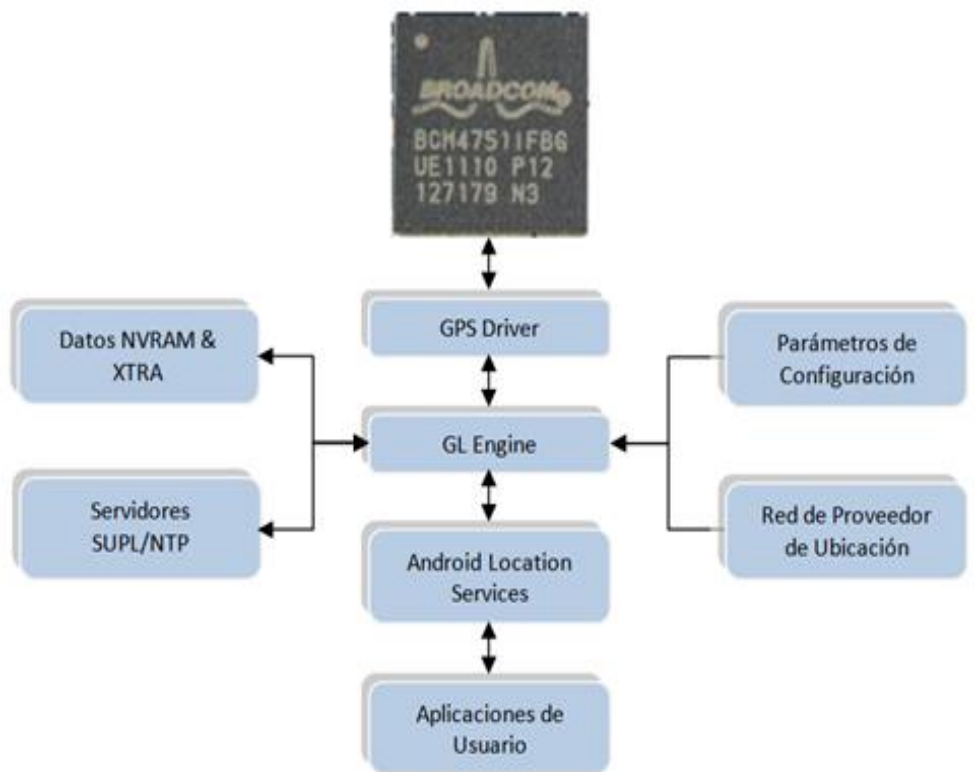

\section{METODOLOGÍA}

Para la aplicación de la técnica NTRIP, se utilizó como receptor base, la estación GNSS de monitoreo continuo Trimble NetR5. Esta generó información de sentencias NMEA (latitud, longitud, altura elipsoidal, hora UTC) (SIRF, 2005) y correcciones de código mediante mensajes RTCM, con un intervalo de un segundo, que fueron enviadas al receptor móvil vía IP. Como móvil se usó el receptor Mobile Mapper10; y una Tablet Samsung Galaxy Tab2 con chip GPS y con Android 4.0.3, que genera información de navegación a partir de sentencias NMEA, que pueden ser descargadas en tiempo real desde una interfaz de programación para Android.

Para realizar la corrección diferencial por posición al móvil, se consideró: la distancia de la base al móvil, que exista cobertura de telefonía celular, y que sean los mismos satélites rastreados tanto en la base como en el móvil. La Figura 3, muestra la arquitectura general utilizada para la aplicación de la técnica NTRIP. 
Figura 3 - Arquitectura General de la Aplicación.

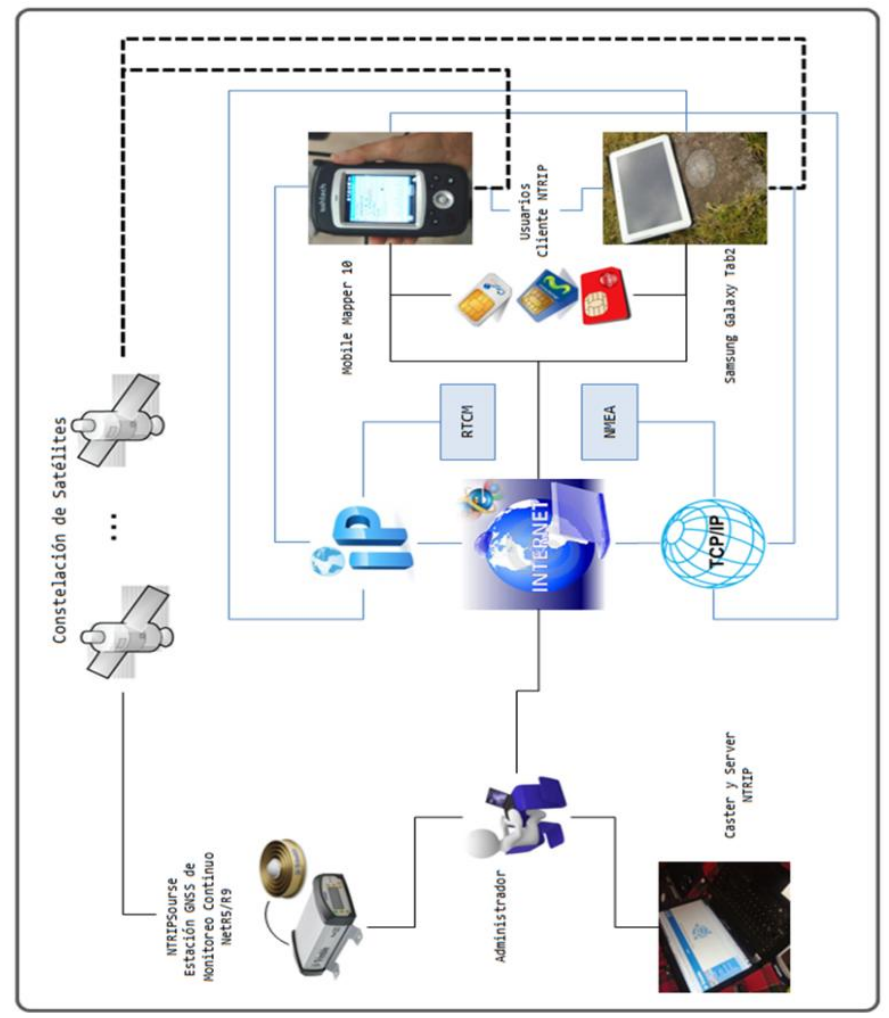

Los equipos móviles tienen limitaciones para poder corregir a partir de las falsas distancias (Pseudoranges), por lo que se procedió a utilizar una Corrección Diferencial por Posición enviada del receptor base, hacia el móvil para mejorar su posición. Esta técnica consiste en calcular las coordenadas de cada posición en el receptor base, y es comparada con las coordenadas conocidas de la misma estación, generando así componentes de corrección en X, Y, Z. En los mismos instantes de tiempo se determina las coordenadas en el móvil con la finalidad de que tengan los mismos satélites rastreados, y poder recibir las correcciones desde la base (DALDA et al., 2008).

Las coordenadas cartesianas de precisión del punto de referencia, son el punto de partida para el cálculo de la corrección, pues las coordenadas marcadas cada instante junto con las coordenadas reales del equipo, no serán las mismas, permitiendo establecer una diferencia de posición en las tres componentes (Ver Figura 4). 
Figura 4 - Punto GPS marcado en un instante $t$ en la Estación Base (Dato NMEA).

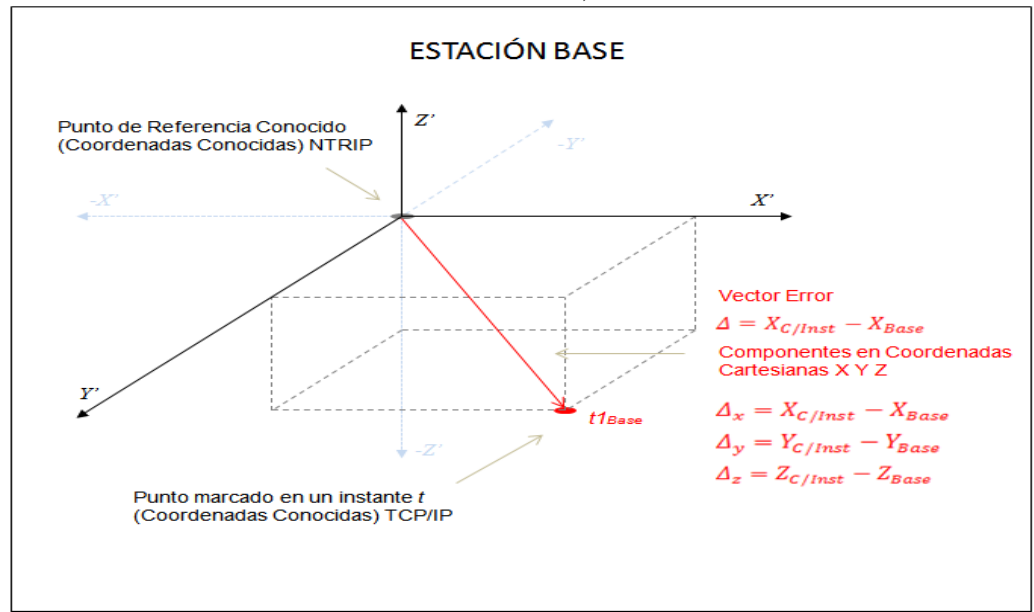

Estas diferencias $\left(\Delta_{X}, \Delta_{Y}, \Delta_{Z}\right)$ deben ser enviadas al móvil (Tablet), para que corrija su posición actual $\left(X_{T A B}, Y_{T A B}, Z_{T A B}\right)$ y obtenga su posición corregida $\left(X_{C O R R}\right.$, $\left.Y_{C O R R}, Z_{C O R R}\right)$ (Ver Figura 5).

Figura 5 - Corrección aplicada al punto original de la Tablet.

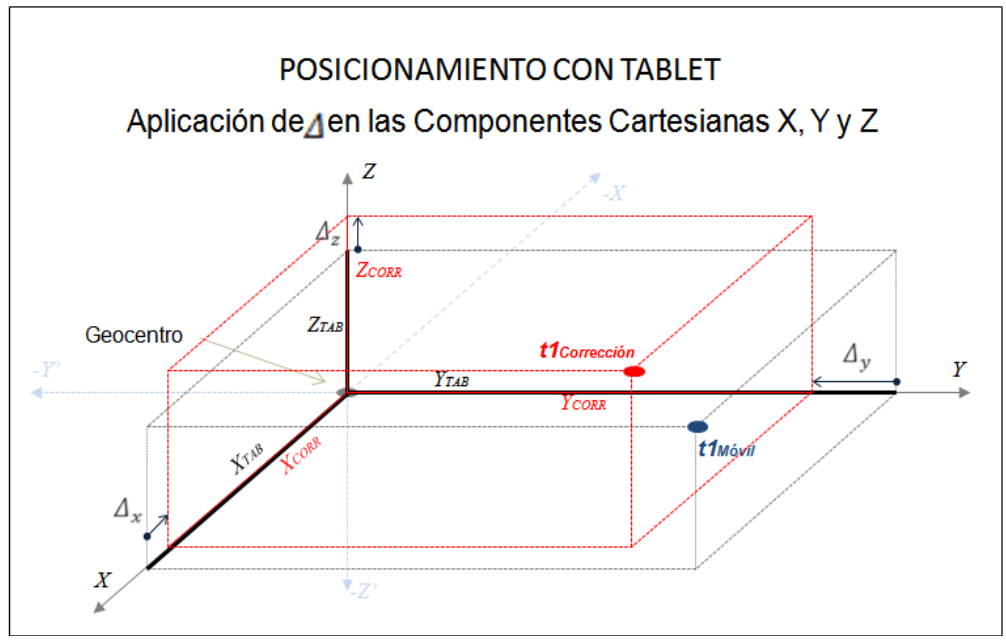

Para obtener las correcciones diferenciales desde la base, y corregirlas en la Tablet en tiempo real fueron desarrollados Interfaces de Programación para 
Android (API, por sus siglas en inglés). Los APIs fundamentales desarrollados para la aplicación NTRIP son indicados a continuación:

- API de ubicación, para tener acceso inmediato al servicio de localización (LocationManager), y activar a los diferentes proveedores de localización (LocationProvider), en este caso, se activó solo el que capta la señal GPS. En el Cuadro 1, se puede observar su código.

Cuadro 1 - Código Fuente de Ubicación.

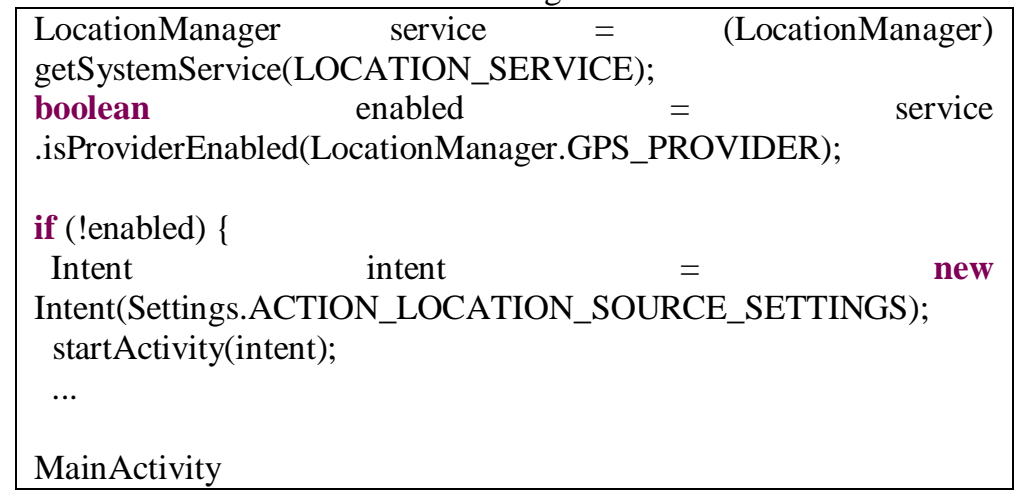

API de Conexiones Inalámbricas, para descargar información desde la estación GNSS de monitoreo continuo, la aplicación debe tener un acceso a internet del tipo 3G o HSPA. El Cuadro 2, se muestra su código.

Cuadro 2 - Código Fuente de Conexiones Inalámbricas.

<?xml version="1.0" encoding="utf-8"?>
<manifest
xmlns:android="http://schemas.android.com/apk/res/android"
package="com. especdp.ntripclient"
android:versionCode="20130325"
android:versionName="2013.03.25"
android:installLocation="auto">
$\quad$ <uses-permission
android:name="android.permission.INTERNET" />
$\quad$ <uses-permission
android:name="android.permission.ACCESS_NETWORK" />
$\quad \ldots$
</application>
$\ldots$
</manifest>




\section{PRUEBAS Y RESULTADOS}

Se realizaron las pruebas respectivas tanto en una Tablet como en Mobile Mapper. La técnica NTRIP se realizó en 8 puntos, en las cuales se conocían las coordenadas respectivas, y que se utilizaron como coordenadas de control, para evaluar el error de posicionamiento del móvil que puede alcanzar después de recibir la corrección diferencial. Las distancias entre la base y el móvil alcanzaron hasta 30 $\mathrm{km}$ aproximadamente. Se realizó un rastreo continuo a cada segundo de intervalo de grabación, y en ese mismo tiempo se recibía las correcciones diferenciales desde el NTRIPCASTER.

Las coordenadas finales del móvil fueron transformadas a un sistema local, y se calcularon las coordenadas planas Este y Norte $(\mathrm{E}, \mathrm{N})$ con la finalidad de determinar la distancia. Se consideró solamente la posición horizontal porque que en vertical los errores son mayores, más aún cuando estamos utilizando la señal de código C/A. Fueron calculadas las diferencias en distancia comparadas con las obtenidas en los puntos de prueba (control). Los resultados obtenidos se pueden observar en la Tabla 1 para la Tablet, y en la tabla 2 para el Mobile Mapper.

Tabla 1 - Resultados con la TABLET.

\begin{tabular}{c|c|c|c|c}
\hline \multirow{2}{*}{$\begin{array}{c}\text { Puntos de } \\
\text { Control }\end{array}$} & \multirow{2}{*}{$\begin{array}{c}\text { Distancia } \\
(\mathbf{K m})\end{array}$} & $\begin{array}{c}\text { NORTE } \\
(\mathbf{m})\end{array}$ & $\begin{array}{c}\text { ESTE } \\
(\mathbf{m})\end{array}$ & $\begin{array}{c}\text { ERROR EN } \\
\text { DISTANCIA } \\
(\mathbf{m})\end{array}$ \\
\hline $\begin{array}{c}\text { CANCHA } \\
\text { ESPE }\end{array}$ & 0,28 & 1,835 & -3.332 & 3,804 \\
\hline CIE04 & 0,47 & $-2,403$ & 0,002 & 2,403 \\
\hline $\begin{array}{c}\text { SANTA } \\
\text { ROSA }\end{array}$ & 2,40 & 0,981 & 1,647 & 1,917 \\
\hline MIRAVALLE & 8,50 & $-1,623$ & 2,513 & 2,991 \\
\hline VICENTINA & 11,86 & $-0,893$ & $-0,172$ & 0,909 \\
\hline H. DEL SUR & 13,10 & 0,750 & $-1,179$ & 1,397 \\
\hline PE-18483-Y & 16,00 & $-0,843$ & 2,520 & 2,657 \\
\hline PE-16943-Y & 31,10 & $-0,270$ & 2,091 & 2,109 \\
\hline $\begin{array}{c}\text { Media } \\
\text { Aritmética }\end{array}$ & & & & 2,273 \\
\hline $\begin{array}{c}\text { Desviación } \\
\text { Estándar }\end{array}$ & & & & 0,910 \\
\hline
\end{tabular}

En la Tabla 1, en la última columna se muestra el error en la distancia horizontal obtenida en la Tablet, mediante la comparación con las distancias obtenidas en los puntos de control a partir de las coordenadas Este y Norte. Los resultados muestran que se puede llegar a tener errores en posicionamiento como media de $2.3 \mathrm{~m}$ aproximadamente. Mientras que en la tabla2, se muestra los 
resultados obtenido con el Mobile Mapper, y puede alcanzar errores medios de 1.6 $\mathrm{m}$ aproximadamente.

Tabla 2 - Resultados con el Mobile Mapper.

\begin{tabular}{c|c|c|c|c}
\hline \multirow{2}{*}{$\begin{array}{c}\text { Puntos de } \\
\text { Control }\end{array}$} & $\begin{array}{c}\text { Distancia } \\
(\mathbf{K m})\end{array}$ & $\begin{array}{c}\text { DORTE } \\
(\mathbf{m})\end{array}$ & $\begin{array}{c}\text { ESTE } \\
(\mathbf{m})\end{array}$ & $\begin{array}{c}\text { DISTANCIA } \\
(\mathbf{m})\end{array}$ \\
\hline $\begin{array}{c}\text { CANCHA } \\
\text { ESPE }\end{array}$ & 0,28 & 0,462 & 0,145 & 0,484 \\
\hline CIE04 & 0,47 & $-0,739$ & $-0,110$ & 0,747 \\
\hline $\begin{array}{c}\text { SANTA } \\
\text { ROSA }\end{array}$ & 2,40 & $-1,258$ & $-1,090$ & 1,665 \\
\hline MIRAVALLE & 8,50 & $-2,192$ & 2,291 & 3,171 \\
\hline VICENTINA & 11,86 & $-1,278$ & 0,884 & 1,554 \\
\hline H. DEL SUR & 13,10 & 1,096 & 0,183 & 1,111 \\
\hline PE-18483-Y & 16,00 & 0,805 & 2,055 & 2,207 \\
\hline PE-16943-Y & 31,10 & 0,065 & 1,713 & 1,715 \\
\hline $\begin{array}{c}\text { Media } \\
\text { Aritmética }\end{array}$ & & & & 1,582 \\
\hline $\begin{array}{c}\text { Desviación } \\
\text { Estándar }\end{array}$ & & & & 0,850 \\
\hline
\end{tabular}

\section{CONCLUSIONES}

- La corrección diferencial (DGPS en tiempo real) que se utilizó en el presente estudio, por posición, tienen las limitantes de distancia, pues a medida que el móvil se aleja de la base, los errores que afectan a las observaciones del receptor móvil difieren de los errores que afectan a las observaciones de la estación base. Los mejores resultados se obtuvieron en un radio de hasta $30 \mathrm{~km}$ con base en la estación GNSS.

- Para que el método de corrección diferencial por posición sea aplicado de la mejor forma, se debe utilizar únicamente la navegación por satélites GPS, dado que los dispositivos móviles tienen varias opciones para calcular la posición, como son las redes y antenas de telefonía celular y la otra por medio de GOOGLE Maps que triangula las coordenadas por medio del internet. Si estas tres opciones están activadas, se produce una respuesta mucho más rápida pero una solución de posición con un mayor error pudiendo llegar a 30 $\mathrm{m}$, pero si solo se activa para recibir la señal de los satélites del GPS baja a $5 \mathrm{~m}$. 
- En un dispositivo móvil activando solo la señal GPS y recibiendo la corrección diferencial vía IP, desde una estación de monitoreo continuo y hasta una distancia de $30 \mathrm{~km}$, se puede obtener errores en la posición horizontal de $2.6 \mathrm{~m}$ con la Tablet y $1.6 \mathrm{~m}$ con el Mobile Mapper 10.

\section{AGRADECIMIENTOS:}

Expresamos nuestro agradecimiento a CEDIA (Consorcio Ecuatoriano para el Desarrollo de Internet Avanzado) quién apoyo con el financiamiento para la ejecución de este proyecto. Así como, a los integrantes de la ESPOCH y de la UTN por su colaboración.

\section{REFERENCIAS BIBLIOGRÁFICAS}

BÁEZ, M., BORREGO, A., CORDERO, J., CRUZ, L., GONZÁlEZ, M., HERNÁNDEZ, F., ZAPATA, A. Introducción al Android. Tecnología UCM. 2010. www.tecnologíaUCM.es, ingresado en 30 de agosto de 2013.

BKG. Networked Transport of RTCM via Internet Protocol, 2005. Ingresado en 15 de noviembre de 2013. http://igs.bkg.bund.de/ntrip.

COSTA, S., LIMA, M., DE MOURA, N., ABREU, M., DA SILVA, A., FORTES, L., MOREIRA, A. RBMC in Real Time Via NTRIP and its Benefits in RTK and DGPS Surveys. Reunión SIRGAS. Buenos Aires, Noviembre 2009 en www.sirgas.org. Ingresado en 20 de noviembre de 2013.

DALDA, A., GONZÁLEZ, F., CANO, M., SÁNCHEZ, J., \& PÉREZ, M. Curso Avanzado de Posicionamiento por Satélite. Instituto Geográfico nacional Ministerio de Fomento. Madrid: Universidad Politécnica de Madrid. 2008.

FERNÁNDEZ, A. Diseño e Implementación de un Generador y un Servidor de Correcciones de GPS Diferencial en Formato RTCM sobre TCP/IP, Mediante una Arquitectura Distribuida. Madrid - España. 2008

FETECUA, H. Implementación de la Red en Panamá GEO-UTP EN Tiempo Real Vía NTRIP. Reunión SIRGAS. Ciudad de Panamá, Octubre 2013 en www.sirgas.org. Ingresado en 18 de marzo de 2014.

HOYER, M., PÉREZ, R., DA COSTA, S., CIOSE, V., CIMBARO, S., NOGUERRA, G., REZZA, R. Avances en la Materealización del Marco de Referencia SIRGAS en Tiempo Real Mediante NTRIP. Reunión SIRGAS. Lima, Noviembre 2010 en www.sirgas.org. Ingresado en 15 de noviembre de 2013.

LATORRE, A. Como está estructurado el GPS en Android. 2012. Ingresado en 5 de octubre de 2013. http://www.elandroidlibre.com

MÁRQUEZ, A. NTRIP Herramienta Indispensable para la Cartografía y el Catastro. II Jornadas Nacionales de Geomática. MECINCA. CaracasVenezuela, 2007.

NILSSON, H. Trends in Functional Programming. Nottingham.2007. 
PIÑON, D., CIMBARO, S. Envío de Correcciones Diferenciales en Tiempo Real a través de Internet. Reunión SIRGAS. Lima, Noviembre 2010 en www.sirgas.org. Ingresado en 10 de noviembre de 2013.

RADIO TECHNICAL COMISSION FOR MARINE SEVICES -RTCM. RTCM Recommended Standard for Differential GNSS (Global Navigation Satellite System) Service Version 2.3. Radio Technical Commission for Maritime Services. Virginia: RTCM. 2001.

SEEBER, G. Satellite Geodesy: Foundations, Methods, and Applications. Walter de Gruyter. New York, 1993.

SILVA, O., Leiva, C., Tierra, A. Implementación de Correcciones Diferenciales en Tiempo Real en Dispositivos Móviles Android con El Uso de NTRIP. Reunión SIRGAS. Ciudad de Panamá, Octubre 2013 en www.sirgas.org.

SIRF. National Marine Electronics Association: NMEA Reference Manual. USA. 2005

TORGE, W; MULLER, J. Geodesy. De Gruyter. 4 Edition, 2012.

YELICICH, R., CAMISAY,M., STRIEWE, E., PÉREZ, R., MACKERN, M. Análisis del Posicionamiento GNSS a Partir de Soluciones de Red(FKP,VRS) obtenidas por NTRIP-RTK en Uruguay. Reunión SIRGAS. Ciudad de Panamá, Octubre 2013 en www.sirgas.org. Ingresado en 20 enero de 2014.

(Recebido em setembro de 2014. Aceito em novembro de 2014). 